\title{
I-Optimal Design of Mixture Experiments in the Presence of Ingredient Availability Constraints
}

\author{
Utami Syafitri*1,2, Bagus Sartono ${ }^{\dagger 2}$ and Peter Goos ${ }^{\ddagger 1,3}$ \\ ${ }^{1}$ Faculty of Applied Economics \& StatUa Center for Statistics, Universiteit Antwerpen, Belgium \\ ${ }^{2}$ Department of Statistics, Bogor Agricultural University, Indonesia \\ ${ }^{3}$ Faculty of Bioscience Engineering \& Leuven Statistics Research Centre, Katholieke Universiteit Leuven, Belgium
}

January 16, 2015

\begin{abstract}
Mixture experiments usually involve various constraints on the proportions of the ingredients of the mixture under study. In this paper, inspired by the fact that the available stock of certain ingredients is often limited, we focus on a new type of constraint, which we refer to as an ingredient availability constraint. This type of constraint substantially complicates the search for optimal designs for mixture experiments. One difficulty, for instance, is that the optimal number of experimental runs is not known a priori. To deal with this complication, we propose a variable neighborhood search algorithm to find I-optimal designs for mixture experiments in case there is a limited stock of certain ingredients.
\end{abstract}

Keywords: variable neighborhood search algorithm, V-optimality.

\section{Introduction}

A mixture experiment is an experiment in which the experimental factors are ingredients of a mixture, and the response depends only on the relative proportions of the ingredients. The proportions of the ingredients all lie between zero and one, and the sum of all ingredient proportions is one. These constraints define a simplex-shaped experimental region: in the absence of any other constraints, a mixture experiment involving $q$ ingredients has a $(q-1)$-dimensional simplex as its experimental region. When there are three ingredients, the experimental region is an equilateral triangle. When there are four ingredients, it is a regular tetrahedron. Standard textbooks on the design of mixture experiments (Cornell, 2002, Smith, 2005) pay much attention to experimental designs specific for simplex-shaped experimental regions and proposed by Scheffé (1958, 1963).

Commonly, at least some of the ingredients in a mixture experiment have lower and/or upper bounds on their proportions. Moreover, there may be multi-component constraints that impose bounds on linear or nonlinear combinations of the ingredient proportions (Cornell, 2002, Smith, 2005, Atkinson, Donev and Tobias, 2007). In that case, it is necessary to compute mixture experimental designs that are optimal

\footnotetext{
${ }^{*}$ Mrs. Utami Syafitri is a predoctoral researcher at the University of Antwerp. Her email address is syafitri.utamidyah@uantwerpen.be

${ }^{\dagger}$ Dr. Sartono is Assistant Professor at the Department of Statistics of Bogor Agricultural University. His email address is bagusco@ipb.ac.id.

${ }_{\ddagger}$ Dr. Goos is Full Professor at the Faculty of Applied Economics and StatUa Center for Statistics of the Universiteit Antwerpen and at the Faculty of Bioscience Engineering and the Leuven Statistics Research Centre of the University of Leuven. He is a Senior Member of the ASQ. His email address is peter.goos@biw.kuleuven.be (corresponding author).
} 
given all constraints on the ingredient proportions. A coordinate-exchange algorithm for the construction of D-optimal mixture designs was proposed by Piepel, Cooley and Jones (2005).

A key feature of all published work on the design of mixture experiments is that it implicitly assumes that the stock of each ingredient is large enough to run every experimental design under consideration. However, in some situations, only a limited stock is available for some of the ingredients. This may render classical designs or optimal designs infeasible. De Ketelaere, Goos and Brijs (2011) discuss a mixture experiment involving flours, and mention that a follow-up experiment was considered but that the availability of certain types of flour for a follow-up experiment was limited. This was due to the fact that the researchers made a major effort to acquire a certain amount of specific flours for the initial experiment. Ordering additional amounts of the same flours was no longer possible by the time the follow-up experiment was planned.

Therefore, in this paper, we study the impact of availability constraints on I-optimal designs for mixture experiments. We study I-optimal mixture designs because the I-optimality criterion, which seeks to minimize the average prediction variance, is suitable when the goal is to optimize one or more responses. This is generally the case for mixture experiments. Also, selecting mixture designs based on the prediction variance was already suggested by Scheffé (1958). A final reason to utilize the I-optimality criterions is that Hardin and Sloane (1993), Goos and Jones (2011) and Jones and Goos (2012) demonstrated that I-optimal response surface designs usually perform well in terms of D-optimality, whereas D-optimal designs usually do not perform well in terms of I-optimality. Remarkably, few researchers have taken up the challenge to seek I-optimal mixture designs. Exceptions are Lambrakis (1968a,b), Laake (1975) and Liu and Neudecker (1995), who presented a limited number of theoretical results, and Goos, Jones and Syafitri (2013), who provide a literature review on I-optimal mixture designs and propose new I-optimal designs for simplexshaped experimental regions. None of the published work on I-optimal mixture designs deals with ingredient availability constraints.

A remarkable feature of the problem of designing mixture experiments in the presence of availability constraints is that the optimal number of runs is not necessarily known beforehand. Imagine a mixture experiment involving several flours, where only $1 \mathrm{~kg}$ is available of a certain ingredient. In that case, it is possible to perform just one experimental run that utilizes all of the ingredient. However, it is also possible to perform two experimental runs using $0.5 \mathrm{~kg}$ each, or one run using $0.2 \mathrm{~kg}$, another run using $0.3 \mathrm{~kg}$ and a final run using $0.5 \mathrm{~kg}$. As a result, finding an optimal experimental design in the presence of availability constraints requires determining the optimal number of runs and the ingredient proportions at each of the runs simultaneously. In this paper, we use a variable neighborhood search algorithm for finding optimal mixture designs in the presence of availability constraints.

The paper is organized as follows. In Section 2, we describe the most commonly used models for data from mixture experiments as well as the I-optimality criterion for selecting designs for mixture experiments. In Section 3, we provide a formal description of the optimal design problem in the presence of ingredient availability constraints. In Section 4, we propose a variable neighborhood search (VNS) algorithm for the I-optimal design of mixture experiments involving availability constraints. In Section 5, we present I-optimal designs for several scenarios involving ingredient availability constraints. We end the paper with a summary.

\section{Models and designs for mixture experiments}

\subsection{Models for data from a mixture experiment}

The most commonly used models for data from mixture experiments are the Scheffé models. If we denote the response measured by $Y$, the number of ingredients in the experiment by $q$ and the proportions of each of the $q$ ingredients by $x_{1}, x_{2}, \ldots, x_{q}$, then the first-order Scheffé model is given by

$$
Y=\sum_{i=1}^{q} \beta_{i} x_{i}+\epsilon
$$


while the second-order Scheffé model is given by

$$
Y=\sum_{i=1}^{q} \beta_{i} x_{i}+\sum_{i=1}^{q-1} \sum_{j=i+1}^{q} \beta_{i j} x_{i} x_{j}+\epsilon .
$$

In these models, $Y$ represents the response variable, the coefficients $\beta_{i}$ represent the expected response when the proportion of the $i$ th ingredient equals 1 , the parameters $\beta_{i j}$ represent the nonlinear blending properties of the $i$ th ingredient and the $j$ th ingredient, and $\epsilon$ represents the random error. A short-hand notation of the two models is

$$
Y=\mathbf{f}^{\prime}(\mathbf{x}) \boldsymbol{\beta}+\epsilon
$$

where $\mathbf{x}$ is the vector containing the ingredient proportions $x_{1}, x_{2}, \ldots, x_{q}, \mathbf{f}(\mathbf{x})$ is the model expansion of $\mathbf{x}$, and $\boldsymbol{\beta}$ is the vector containing the model's coefficients $\beta_{i}$ and, for the second-order model, $\beta_{i j}$. For the firstorder Scheffé model, $\mathbf{f}(\mathbf{x})=\mathbf{x}$, while, for the second-order Scheffé model, it is $\mathbf{f}(\mathbf{x})=\left(\mathbf{x}^{\prime}, x_{1} x_{2}, x_{1} x_{3}, \ldots\right.$, $\left.x_{q-1} x_{q}\right)^{\prime}$. The dimension of $\mathbf{f}(\mathbf{x})$ and $\boldsymbol{\beta}$ is denoted by $p$ in the remainder of this paper. It equals $q$ for first-order models and $q(q+1) / 2$ for second-order models.

In matrix notation, the models in Equations (1), (2) and (3) can be written as

$$
\mathbf{Y}=\mathbf{X} \boldsymbol{\beta}+\boldsymbol{\epsilon}
$$

where $\mathbf{Y}$ is the $n$-dimensional vector of responses, $\mathbf{X}$ represents the $n \times p$ model matrix containing the settings and the model expansions of the mixture ingredient proportions $x_{1}, \ldots, x_{q}$, for each of the $n$ runs of the experiment, and $\boldsymbol{\epsilon}$ is an $n$-dimensional vector containing the random errors of the individual runs. We assume that $\boldsymbol{\epsilon}$ is normally distributed with zero mean and variance-covariance matrix $\sigma_{\epsilon}^{2} \mathbf{I}_{n}$. In that case, the best linear unbiased estimator of the coefficient vector $\boldsymbol{\beta}$ is the ordinary least squares estimator

$$
\hat{\boldsymbol{\beta}}=\left(\mathbf{X}^{\prime} \mathbf{X}\right)^{-1} \mathbf{X}^{\prime} \mathbf{Y}
$$

\subsection{Constrained mixture experiment}

Mixture experiments are often characterized by the presence of constraints on the ingredient proportions. Simple, common constraints are lower and upper bounds on the proportions of the ingredients. In general, these constraints can be written as

$$
0 \leq L_{i} \leq x_{i} \leq U_{i}
$$

where $L_{i}$ represents the lower bound for the proportion of ingredient $i$ and $U_{i}$ represents the upper bound. In case lower bounds on individual ingredient proportions are the only constraints, the experimental region remains a regular simplex. Kurotori (1966) suggested transforming the constrained ingredient proportions into coded values called $L$-pseudocomponents. The coded value of the $i$ th ingredient proportion is

$$
x_{i}^{*}=\frac{x_{i}-L_{i}}{1-\sum_{i=1}^{q} L_{i}} .
$$

Because the pseudocomponents are linear transformations of the original ingredient proportions, finding an Ioptimal design expressed in terms of pseudocomponents is mathematically equivalent to finding an I-optimal design expressed in terms of the original ingredient proportions (Atkinson, Donev and Tobias, 2007).

\subsection{Simplex-lattice designs for mixture experiments}

A $\{q, m\}$ simplex-lattice design consists of all combinations of the proportions $\{0,1 / m, 2 / m, \ldots, 1\}$ for the $q$ ingredients whose proportions sum to one. The simplex-lattice design involves

$$
\left(\begin{array}{c}
m+q-1 \\
m
\end{array}\right)
$$


different combinations or design points. For example, a $\{3,1\}$ simplex-lattice design for three ingredients involves three design points, $(1,0,0),(0,1,0)$, and $(0,0,1)$. As each design point requires $100 \%$ of one ingredient, the design points correspond to the pure components. A $\{3,2\}$ simplex-lattice design consists of six design points, $(1,0,0),(0,1,0),(0,0,1),(0,1 / 2,1 / 2),(1 / 2,1 / 2,0)$, and $(1 / 2,0,1 / 2)$. The first three design points are pure components. The last three design points are binary mixtures since they involve nonzero proportions of only two ingredients. In this paper, some designs involve ternary and quaternary mixtures, i.e. where there are three or four nonzero ingredient proportions. Often, this results from the use of non-zero lower bounds on all components.

Any $\{q, 1\}$ simplex-lattice design involves the $q$ vertices of the experimental region, whereas any $\{q, 2\}$ simplex-lattice design involves the $q$ vertices and $q(q-1) / 2$ binary mixtures involving $50 \%$ of one ingredient and $50 \%$ of another. These two types of designs were associated with the first- and second-order Scheffé model, respectively (Scheffé, 1958). Simplex-lattice designs can be constructed for any simplex-shaped experimental region, either in the original ingredients (if there are no lower bounds on the ingredient proportions) or in the pseudocomponents (in case there are lower bounds on the ingredient proportions). Note that, if there is a nonzero lower bound on each ingredient proportion, a simplex-lattice design in the pseudocomponents involves the points $(1,0,0),(0,1,0)$, and $(0,0,1)$, but these points correspond in fact to ternary mixtures.

\subsection{I-optimality criterion}

Rather than on the precise estimation of the parameter vector $\boldsymbol{\beta}$, the I-optimality criterion focuses on precise prediction throughout the entire experimental region. An I-optimal design minimizes the average prediction variance

$$
\frac{\int_{S_{q-1}} \sigma^{2} \mathbf{f}^{\prime}(\mathbf{x})\left(\mathbf{X}^{\prime} \mathbf{X}\right)^{-1} \mathbf{f}(\mathbf{x}) d \mathbf{x}}{\int_{S_{q-1}} d \mathbf{x}}
$$

which can be rewritten as

$$
\frac{1}{\int_{S_{q-1}} d \mathbf{x}} \sigma^{2} \operatorname{tr}\left[\left(\mathbf{X}^{\prime} \mathbf{X}\right)^{-1} \mathbf{B}\right]
$$

where $S_{q-1}$ represents the experimental region and

$$
\mathbf{B}=\int_{S_{q-1}} \mathbf{f}(\mathbf{x}) \mathbf{f}^{\prime}(\mathbf{x}) d \mathbf{x}
$$

is the moments matrix.

In case the experimental region is a $(q-1)$-dimensional simplex, the individual elements of the moments matrix can be calculated using the expression

$$
\int_{S_{q-1}} x_{1}^{p_{1}-1} x_{2}^{p_{2}-1} \ldots x_{q}^{p_{q}-1} d x_{1} d x_{2} \ldots d x_{q-1}=\frac{\prod_{i=1}^{q} \Gamma\left(p_{i}\right)}{\Gamma\left(\sum_{i=1}^{q} p_{i}\right)}
$$

which was given, for instance, in De Groot (1970). The volume of a $(q-1)$-dimensional simplex in the absence of lower bounds on the ingredient proportions is

$$
\int_{S_{q-1}} d \mathbf{x}=\frac{1}{\Gamma(q)} .
$$

For a given design problem, the volume of the experimental region is constant. It is therefore irrelevant when it comes to finding an I-optimal design, as is $\sigma^{2}$.

When comparing the performance of two designs with model matrices $\mathbf{X}_{1}$ and $\mathbf{X}_{2}$ in terms of the Ioptimality criterion, we use the relative I-efficiency

$$
\frac{\operatorname{tr}\left[\left(\mathbf{X}_{2}^{\prime} \mathbf{X}_{2}\right)^{-1} \mathbf{B}\right]}{\operatorname{tr}\left[\left(\mathbf{X}_{1}^{\prime} \mathbf{X}_{1}\right)^{-1} \mathbf{B}\right]} .
$$


This relative efficiency takes a value larger than one if the design with model matrix $\mathbf{X}_{1}$ outperforms the one with model matrix $\mathbf{X}_{2}$ in terms of average prediction variance.

A review and some new results on I-optimal designs for Scheffé models in the absence of ingredient availability constraints are given in Goos, Jones and Syafitri (2013) and Goos and Syafitri (2014). Note that the I-optimality criterion is sometimes also named the V-optimality criterion.

\subsection{Fraction of design space plot}

The performance of an experimental design in terms of the prediction variance can be visualized by constructing a fraction of design space (FDS) plot, which shows the cumulative distribution of the predicton variance scaled by $\sigma^{2}$ :

$$
\mathbf{f}^{\prime}(\mathbf{x})\left(\mathbf{X}^{\prime} \mathbf{X}\right)^{-1} \mathbf{f}(\mathbf{x})
$$

across the entire experimental region or design space. The FDS plot allows us to see the minimum and maximum prediction variance, as well as any percentile of the prediction variance. It therefore provides a more detailed picture of a design's performance in terms of the prediction variance than the average prediction variance (i.e., the I-optimality criterion). Ideally, an experimental design leads to a small prediction variance in large portions of the experimental region.

\section{The problem}

In this section, we discuss the optimization problem that needs to be solved to obtain an I-optimal mixture design in the presence of ingredient availability constraints. A key feature of our approach to solving that problem is that it involves a set of possible design points, called a candidate set and consisting of combinations of feasible ingredient proportions. In any feasible design for a mixture experiment, each of these candidate design points is either used or not. In case a given candidate point is used in the design, it can be used once or more than once. We denote the $c$ th candidate point by $\mathbf{x}_{c}=\left(x_{1 c}, x_{2 c}, \ldots, x_{q c}\right)$ and the number of times the candidate point appears in the design by $z_{c}$, where $z_{c} \in\{0,1,2, \ldots\}$.

The challenge is to identify the values of $z_{c}$ that result in an optimal value for the I-optimality criterion without violating the availability constraints for each of the ingredients. If we denote the number of candidate points by $N$, then the number of experimental runs corresponding to a given solution for the $z_{c}$ values equals

$$
n=\sum_{c=1}^{N} z_{c}
$$

If we denote the availability of the $i$ th ingredient by $R_{i}$ and the total amount of the mixture required for one experimental run by $\lambda$, the availability constraint for ingredient $i$ can be written as

$$
\lambda \sum_{c=1}^{N} x_{i c} z_{c} \leq R_{i}
$$

There is no explicit upper bound for the number of experimental runs $n$, but the total amount of the $n$ mixtures used in the experiment cannot exceed

$$
R=\sum_{i=1}^{q} R_{i}
$$

An implicit upper bound for $n$ therefore is $R / \lambda$. Consequently, $R / \lambda$ is also the maximum value each $z_{c}$ can take. Without loss of generality, we set $\lambda=1$ in the remainder of this paper.

The objective function of the optimization problem we face can be formulated as

$$
\text { Minimize } \operatorname{tr}\left(\mathbf{X}^{\prime} \mathbf{X}\right)^{-1} \mathbf{B}=\operatorname{tr}\left[\left\{\sum_{c=1}^{N} z_{c} \mathbf{f}\left(\mathbf{x}_{c}\right) \mathbf{f}^{\prime}\left(\mathbf{x}_{c}\right)\right\}^{-1} \mathbf{B}\right]
$$


Readers who are familiar with operations research might observe similarities between the optimization problem we consider here and the well-known knapsack problem (Kellerer, Pferschy and Pisinger, 2010). Indeed, the problem of finding an optimal design for mixture experiments involving availability constraints is a bounded nonlinear, nonseparable multidimensional knapsack problem. As a result thereof, many solution approaches presented in the literature for knapsack problems cannot be used. This is because the approaches for the simplest knapsack problems exploit the linear nature of the objective function and the constraints, those for quadratic knapsack problems also exploit the specific nature of the objective function, and those for nonlinear knapsack problems focus on the subclass of separable nonlinear knapsack problems. Therefore, we resort to a heuristic solution approach. More specifically, we use a variable neighborhood search approach because such an approach has been used successfully to tackle various other hard optimization problems in optimal design of experiments.

Note that, in this article, we consider a scenario in which there is no constraint on the number of runs other than the implicit one imposed by the ingredient availabilities. Thus, we assume that the experimenters want to get the maximum out of the available ingredients. In experimental settings where the cost or the time required to perform an experimental run is large, an explicit upper bound, unrelated to the ingredient availabilities, may be imposed on the number of runs. If the upper bound is small, this may render some or all of the availability constraints moot. The experimental design problem faced by the experimenter then simplifies to one without availability constraints.

\section{Variable neighborhood search algorithm}

\subsection{Main idea}

Variable neighborhood search (VNS) was introduced by Mladenović and Hansen (1997) as an improvement over local-search-based algorithms for combinatorial optimization. In local-search-based algorithms, the search for a good solution is done by iteratively making one small change (called a move) to the current solution $s$. All solutions $s^{\prime}$ that can be reached in one single move starting from a given solution $s$ form the neighborhood of that solution, denoted by $N(s)$. A solution whose neighborhood does not contain any better solution is called a local optimum with respect to the neighborhood. Many heuristic algorithms have been developed, each using a different strategy to escape from these local optima and hopefully reach a globally optimal solution.

Unlike local-search-based algorithms, VNS systematically explores different neighborhood structures (i.e., neighborhoods defined by different types of moves). The main idea of VNS is that a solution that is a local optimum relative to a certain neighborhood structure not necessarily is a local optimum relative to another neighborhood structure. For this reason, escaping from a locally optimal solution can be done by changing the neighborhood structure. VNS has been successfully applied to a wide variety of optimization problems such as vehicle routing (Kytöjoki, Nuortio, Bräysy and Gendreau, 2007), project scheduling (Fleszar and Hindi, 2004), automatic discovery of theorems (Caporossi and Hansen, 2004), graph coloring (Avanthay, Hertz and Zufferey, 2003), and the synthesis of radar polyphase codes (Mladenović, Petrović, Kovačević-Vujčić and Čangalović, 2003). In the field of experimental design, VNS has been used for the determination of optimal run orders for the design points of standard experimental designs in the presence of serially correlated responses (Garroi, Goos and Sorensen, 2010) and to assign treatments to whole plots in orthogonal split-plot designs (Sartono, Goos and Schoen, 2015). In both cases, solving the optimal experimental design problem at hand came down to finding an optimal permutation. This is very different from the optimal design problem considered in this paper, where the optimal number of experimental runs needs to be found, as well as the optimal design points and the number of times each design point is used.

Most implementations of VNS algorithms explore the different neighborhoods in a sequential fashion, starting with the neighborhood that includes the fewest solutions and ending with the neighborhood that contains the largest number of solutions. Larger neighborhoods are only explored when the current solution

is a local optimum with respect to all smaller neighborhoods, to save computing time. Ultimately, a VNS algorithm produces a solution that is a local optimum with respect to all neighborhoods considered. This 
makes it more likely that the solution produced is a globally optimal solution.

\subsection{Our algorithm}

Our VNS algorithm involves two steps. In the first step, a starting design is created. In the second step, different neighborhoods are explored to improve the starting design, so as to turn it into a solution that is a local optimum with respect to all neighborhoods. In this section, we first discuss the input to the algorithm. Next, we present the different neighborhoods we utilize, as well as a detailed description of the algorithm.

\subsubsection{Input}

A key input to our algorithm is the set of mixtures that can be used in the experiment (i.e., the possible design points). In the optimal experimental design literature, this set is usually referred to as the candidate set. We start the construction of the candidate set by considering all points of the $\{q, h\}$ simplex-lattice design, where $q$ denotes the number of ingredients in the mixture and $h$ is a nonzero integer. In case there are lower and upper bounds for the ingredient proportions, and/or other constraints involving more than one ingredient, we drop the simplex-lattice points that do not satisfy the constraints. The remaining points then form the final candidate set.

Ideally, the candidate set is as large as possible, implying that the value of $h$ should be as large as possible. However, for a mixture experiment to be practically feasible, $h$ should not be excessively large. Otherwise, it may be impossible to actually make the mixture with the proportions corresponding to the candidate points. Moreover, there is a decreasing marginal utility for increasing the value of $h$. In other words, the extent to which the I-optimality of a design can be improved by increasing $h$ by one unit becomes smaller and smaller for larger values of $h$. In our examples in the next sections, we set $h$ to 20 .

Besides the candidate set, which we call $\mathcal{C}$ in the remainder of this paper, the input to our algorithm consists of the model specification (first-order, second-order, ...) and the availabilities $R_{1}, R_{2}, \ldots, R_{q}$ of the $q$ ingredients.

\subsubsection{Starting design}

Our algorithm starts by randomly generating an initial design. This is done by randomly selecting points from the candidate set $\mathcal{C}$ until at least one of the ingredient availabilities is exceeded. The final feasible design produced by this procedure is the starting design for the VNS procedure. The number of design points in the starting design is random. The maximum number of points in the starting design depends on the ingredient availabilities and on the bounds for each of the ingredients in the mixture.

\subsubsection{The variable neighborhood search}

Our algorithm improves the starting design by iteratively making small changes to the design. The algorithm considers four types of changes or moves, each of which defines a neighborhood. The first type of move involves adding a point from the candidate set to the design. Therefore, the neighborhood $N_{0}$ of the starting design is the set of all designs that can be obtained from the starting design by adding a point from the candidate set. The point added does not need to be different from the points in the starting design. The second type of move involves replacing a point from the design by a point from the candidate set. This move type defines neighborhood $N_{1}$. The third move type involves replacing a design point with two points from the candidate set and defines neighborhood $N_{2}$. The final type of move, which determines neighborhood $N_{3}$, requires the replacement of two points from the design by two points from the candidate set. In our algorithm, we only consider feasible solutions (i.e., mixture designs that do not violate the availability constraints). Therefore, the neighborhoods in our algorithm only involve feasible designs.

Neighborhood $N_{0}$ is the smallest of the four neighborhoods, while neighborhood $N_{3}$ is the largest. Denoting the number of points in the design under evaluation by $n_{c}$, these two neighborhoods contain at most $N$ and $n_{c}\left(n_{c}-1\right) \times N^{2}$ solutions, respectively. Neighborhoods $N_{1}$ and $N_{2}$ contain at most $n_{c} \times N$ and $n_{c} \times N^{2}$ solutions, respectively. An overview of the four neighborhoods and their maximum sizes is shown in Table 1. 
Table 1: Neighborhood structures $N_{k}$ for the VNS algorithm

\begin{tabular}{lcl}
\hline$N_{k}$ & Size & Description \\
\hline$N_{0}$ & $N$ & Add a candidate point to the current design \\
$N_{1}$ & $n_{c} \times N$ & Remove a current design point and add a candidate point \\
$N_{2}$ & $n_{c} \times N^{2}$ & Remove a current design point and add two candidate points \\
$N_{3}$ & $n_{c}\left(n_{c}-1\right) \times N^{2}$ & Remove two current design points and add two candidate points \\
\hline
\end{tabular}

Our algorithm explores the four neighborhoods, starting with the smallest neighborhood $N_{0}$ and ending with the largest one, $N_{3}$. This means that the algorithm starts by seeking neighboring solutions in neighborhood $N_{0}$ that have a better I-optimality criterion value than the current solution. As soon as such a solution has been found, the neighborhood $N_{0}$ of that newly found solution is constructed and explored to attempt to find an even better solution in terms of the I-optimality criterion. At some point in time, this procedure will result in a solution for which the neighborhood $N_{0}$ does not contain any better solution. That solution then is a local optimum with respect to neighborhood $N_{0}$. It might, however, not be a locally optimal solution with respect to neighborhood $N_{1}$. Therefore, the next step is to explore the neighborhood $N_{1}$ of that solution until a better one is found. As soon as a better solution has been identified in neighborhood $N_{1}$, the algorithm switches back to the smallest neighborhood $N_{0}$ and attempts to find a better solution in that neighborhood. If no better solution can be found in neighborhood $N_{1}$, then the algorithm switches to neighborhood $N_{2}$. Finally, if neighborhood $N_{2}$ does not contain any better solution, neighborhood $N_{3}$ is explored. This process is repeated until a solution is obtained that is a local optimum with respect to all of the neighborhoods $N_{0}-N_{3}$.

Each time an improved solution is found in one of the larger neighborhoods, the algorithm returns to the smallest neighborhood $N_{0}$. This way, the algorithm avoids having to explore the largest neighborhoods oftentimes, thereby limiting its computing time. Note that, when the number of experimental runs in the current design is maximal (because all the available resources have been used), the neighborhoods $N_{0}$ and $N_{2}$ are skipped because it is then no longer possible to add experimental runs to the design. The structure of the VNS algorithm is shown schematically in Figure 1.

Our algorithm is a first-improvement algorithm, since it does not evaluate all solutions contained within a neighborhood. Instead, it evaluates the solutions contained within the neighborhood of a given solution one by one, until a better solution is found. The search process is then re-centered around the newly obtained better solution, and neighborhood $N_{0}$ of that newly obtained solution is investigated.

In order to increase the likelihood of finding a globally optimal mixture design, we repeat the entire search a prespecified number of times, each time starting from a different randomly generated initial solution and using a different random order of the candidate set. The different ordering of the candidate set ensures that the exploration of each neighborhood is performed in a different order at each iteration.

We implemented the VNS algorithm in the SAS procedure iml. We extracted the moments matrices required for the computation of I-optimal designs from the software package JMP.

\section{$5 \quad$ Examples}

In this section, we report the results of our VNS algorithm for five experimental scenarios involving availability constraints. While our algorithm can handle other models as well, we assumed that the interest was in the second-order Scheffé model in each scenario. Some scenarios do not involve any constraints on the ingredient proportions other than the availability constraints. Other scenarios involve only lower bounds, and one example involves both upper bounds and lower bounds. In all scenarios, we assume that every run requires a total of $1 \mathrm{~kg}$ of ingredients.

Given that our algorithm uses a candidate set, it is easy to handle linear and non-linear multicomponent constraints in addition to lower and upper bound constraints. The only thing that is required is that any 


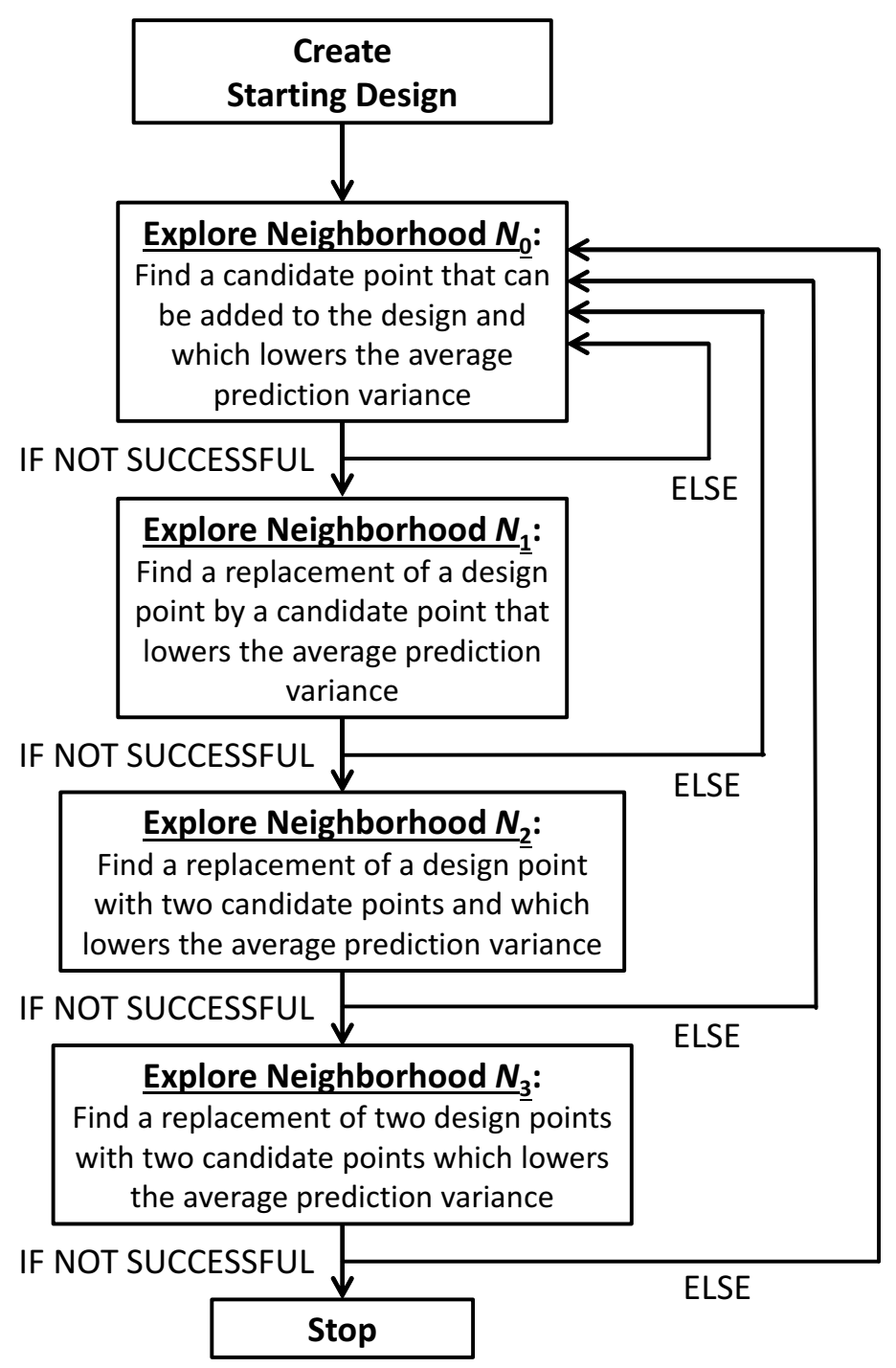

Figure 1: Flow chart of one execution of the variable neighborhood search (VNS) algorithm for finding an I-optimal design in the presence of ingredient availability constraints. 


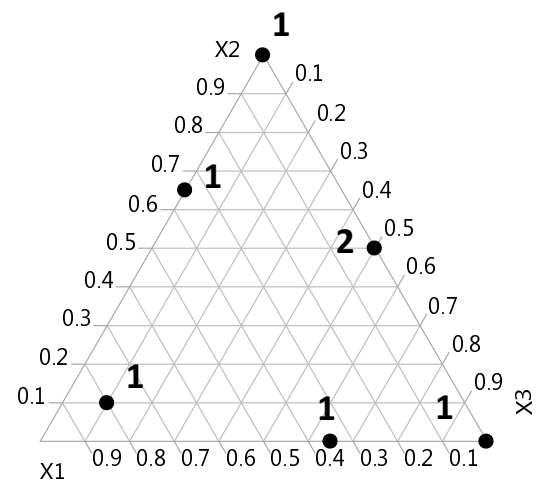

(a) Scenario 1

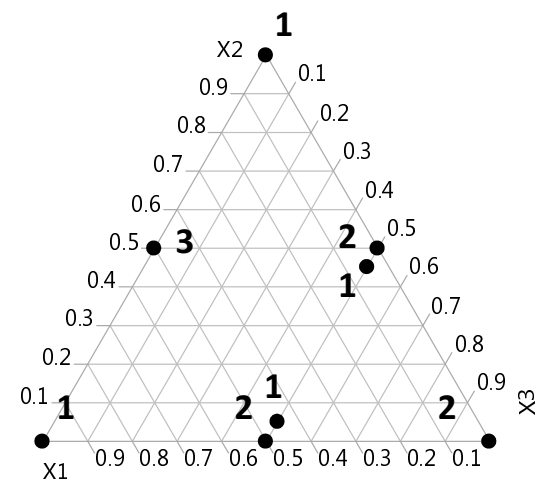

(b) Scenario 2

Figure 2: I-optimal three-ingredient designs for Scenarios 1 and 2.

points that do not satisfy the constraints are removed from the candidate list.

\subsection{Three ingredients}

\subsubsection{Three unconstrained ingredients}

Suppose that we have three ingredients and that there are no lower or upper bounds on the ingredient proportions to be used at each experimental run. In Scenario 1, we assume that the available stock of ingredient 1 is $1.5 \mathrm{~kg}$, the stock of the ingredient 2 is $3 \mathrm{~kg}$, and the stock of the ingredient 3 is also $3 \mathrm{~kg}$. In Scenario 2, we assume that the available stocks are $4 \mathrm{~kg}$ for ingredients 1 and 2, and $5 \mathrm{~kg}$ for ingredient 3 . Figures 2(a) and 2(b) show the I-optimal designs for Scenario 1 and Scenario 2, respectively.

The I-optimal design for Scenario 1 involves six distinct design points: two pure components, three binary mixtures, and a ternary mixture. The I-optimal design does not involve the design point $\left(x_{1}, x_{2}, x_{3}\right)=$ $(1,0,0)$. Instead, it involves a ternary mixture, corresponding to the design point $\left(x_{1}, x_{2}, x_{3}\right)=(0.8,0.1,0.1)$ which uses all three ingredients. Additionally, the I-optimal design involves the design points $\left(x_{1}, x_{2}, x_{3}\right)=$ $(0.35,0.65,0)$ and $\left(x_{1}, x_{2}, x_{3}\right)=(0.35,0,0.65)$. The I-optimum design requires $1.5 \mathrm{~kg}$ of the first ingredient, $2.75 \mathrm{~kg}$ of the second ingredient, and $2.75 \mathrm{~kg}$ of the third ingredient. So, the design utilizes the entire available stock of the first ingredient, which has the smallest availability. The I-optimality criterion value of the design is 0.6700 .

The I-optimal design for Scenario 2 has eight distinct design points instead of six. Six of the eight points correspond to the points of the $\{3,2\}$ simplex-lattice design. The two additional points involve ternary mixtures, $\left(x_{1}, x_{2}, x_{3}\right)=(0.45,0.05,0.5)$ and $\left(x_{1}, x_{2}, x_{3}\right)=(0.05,0.45,0.5)$. The design is shown in Figure 2(b). The I-optimality criterion value of the I-optimal design is 0.2603 . The design requires $4 \mathrm{~kg}$ of the first ingredient, $4 \mathrm{~kg}$ of the second ingredient, and $5 \mathrm{~kg}$ of the third ingredient.

The fact that the I-optimal design for Scenario 2 involves the ternary mixtures $\left(x_{1}, x_{2}, x_{3}\right)=(0.45,0.05,0.5)$ and $\left(x_{1}, x_{2}, x_{3}\right)=(0.05,0.45,0.5)$ may come as a surprise, and may be viewed as an indication that our algorithmic approach to identify optimal designs failed. This is because many researchers would expect only pure components and binary mixtures to appear in an optimal design for the second-order Scheffé model. To investigate this issue, we replaced the ternary mixtures $\left(x_{1}, x_{2}, x_{3}\right)=(0.45,0.05,0.5)$ and $\left(x_{1}, x_{2}, x_{3}\right)=(0.05,0.45,0.5)$ by the binary mixtures $\left(x_{1}, x_{2}, x_{3}\right)=(0.5,0,0.5)$ and $\left(x_{1}, x_{2}, x_{3}\right)=(0,0.5,0.5)$, respectively. The resulting design then has three observations at $\left(x_{1}, x_{2}, x_{3}\right)=(0.5,0,0.5)$ and three observations at $\left(x_{1}, x_{2}, x_{3}\right)=(0,0.5,0.5)$, and it also requires $4 \mathrm{~kg}$ of the first ingredient, $4 \mathrm{~kg}$ of the second ingredient, and $5 \mathrm{~kg}$ of the third ingredient. The design performs slightly worse than the design in Figure 2(b) involving the two ternary mixtures: it results in an I-optimality criterion value of 0.2611 , and has 


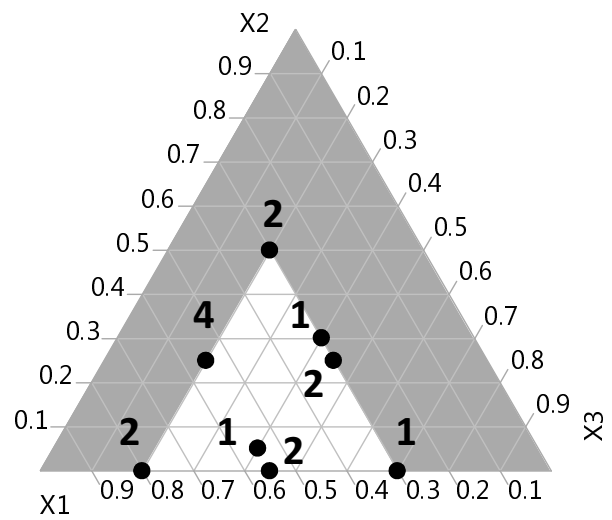

Figure 3: I-optimal design for Scenario 3.

an I-efficiency of $99.71 \%$ relative to the original design.

\subsubsection{Three ingredients with lower bounds}

Figure 3 shows the I-optimal design for Scenario 3, involving a second-order model, lower bounds of 0.3 and 0.2 for the proportions of ingredient 1 and ingredient 3, respectively, and availabilities of $10.2 \mathrm{~kg}, 4 \mathrm{~kg}$ and $4.9 \mathrm{~kg}$ for ingredients 1,2 and 3, respectively. The white triangle in Figure 3 shows the constrained experimental region, which takes into account the lower bounds for ingredients 1 and 3 . The larger grey triangle represents the experimental region in the event there are no lower bounds. Since this experiment only involves lower bounds on the ingredient proportions, the constrained design region is simplex-shaped.

The I-optimal design consists of 15 runs at 8 distinct design points. It is shown in Figure 3 . The design involves ten binary mixtures as opposed to five pure components. Therefore, it does not put much emphasis on the vertices. This is in line with the theoretical results concerning I-optimal designs in the absence of ingredient availability constraints (see Goos, Jones and Syafitri (2013)). The required amounts of the three ingredients equal 7.25, 2.85 and 4.9 for the I-optimal design. The I-optimality criterion value of the 15-run I-optimal design is 0.2323 .

A remarkable fact about the I-optimal design is that it requires only $2.85 \mathrm{~kg}$ of the most scarce ingredient (ingredient 2), which has an availability of $4 \mathrm{~kg}$. Ingredient 3, which has an availability of $4.9 \mathrm{~kg}$, is the only ingredient whose entire stock is used. This is counterintuitive at first sight. However, ingredient 2 does not have a lower bound on its proportion, which implies that many experimental runs can be performed without emptying the stock of that ingredient. Ingredient 3, however, does have a lower bound of 0.2 on its proportion. This implies that every single experimental run requires some of ingredient 3. For this reason, the limited availability of ingredient 3 is more problematic than that of ingredient 2 . In general, it is the ingredients for which the availability is $R_{i}$ small and the lower bound $L_{i}$ is large that will limit the number of runs that can be conducted, and, hence, limit the information content of the experimental design.

\subsubsection{Three ingredients with lower and upper bounds}

Scenario 4 involves lower and upper bounds of the ingredient proportions. The proportion of the first ingredient has to lie between 0.1 and 0.4 . The proportion of the second ingredient has to lie between 0.2 and 0.5 . Finally, the proportion of the third ingredient has to lie between 0.1 and 0.7 . The resulting constrained design region is a parallelogram, as shown by the white area in Figure 4. The available stock of ingredients 1,2 and 3 is $2.5 \mathrm{~kg}, 4 \mathrm{~kg}$, and $10 \mathrm{~kg}$, respectively. We based the candidate set for our algorithm on the 


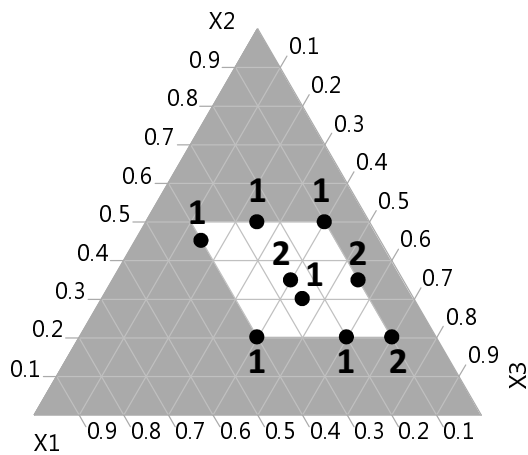

(a) Original I-optimal design for Scenario 4

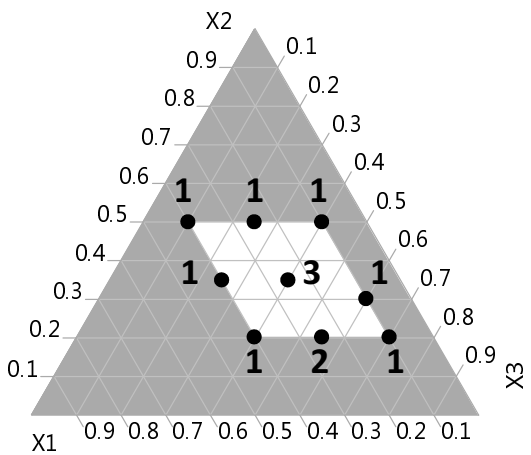

(b) New I-optimal design for Scenario 4

Figure 4: The I-optimal designs for second-order model of Design 4

$\{3,20\}$ simplex-lattice design. After removing the points that did not satisfy the lower and upper bounds, 49 candidate points remained.

The I-optimal design for this scenario involves 12 runs and 9 distinct design points. Figure 4(a) shows the composition of the I-optimal design. Three of the nine design points appear in the design twice. From an optimal design of experiments perspective, a few things are unusual about the I-optimal design. First, the top left vertex of the design region, with coordinates $\left(x_{1}, x_{2}, x_{3}\right)=(0.4,0.5,0.1)$, does not appear in the design. Instead, the point $\left(x_{1}, x_{2}, x_{3}\right)=(0.4,0.45,0.15)$ is a design point. One point, $\left(x_{1}, x_{2}, x_{3}\right)=$ $(0.25,0.3,0.45)$, is right next to the overall centroid of the parallelogram-shaped region, and another point, $\left(x_{1}, x_{2}, x_{3}\right)=(0.2,0.2,0.6)$, is to the right of the centroid of the bottom edge of the design region. The I-optimal design requires $2.5 \mathrm{~kg}$ of the first ingredient, $3.95 \mathrm{~kg}$ of the second ingredient, and $5.55 \mathrm{~kg}$ of the third ingredient. The I-optimality criterion value of the design is 0.3492 .

The fact that the I-optimal design in Figure 4(a) uses the entire stock of the first ingredient might inspire the experimenters to order an additional amount of that ingredient. In case an extra $0.5 \mathrm{~kg}$ is ordered, the total availability of ingredient 1 becomes $3 \mathrm{~kg}$. This leads to a new I-optimal design, involving 12 runs and nine distinct design points. The new I-optimal design is shown in Figure 4(b). The number of distinct design points is the same as in the original I-optimal design in Figure 4(a), but the locations of four of the nine design points differ. One change is that the top left vertex of the design region, with coordinates $\left(x_{1}, x_{2}, x_{3}\right)=(0.4,0.5,0.1)$, now does appear in the design.

The new I-optimal design requires $3 \mathrm{~kg}$ of the first ingredient, $4 \mathrm{~kg}$ of the second ingredient, and $5 \mathrm{~kg}$ of the third ingredient. So, the design exhausts the entire stocks of the first ingredient and the second ingredient.

The I-optimality criterion value of the new design is 0.3101 , versus 0.3492 for the original design. As a result, the I-efficiency of the original I-optimal design relative to the new I-optimal design is $88.80 \%$. Clearly, the new I-optimal design is substantially better than the previous design in terms of average variance of prediction. The fraction of design space plot in Figure 5 shows that the new I-optimal design (represented by the dashed line) is superior to the original I-optimal design (represented by the solid line) in terms of prediction variance in most of the experimental region. The median prediction variances for the two designs are visualized by means of the horizontal dashed lines and equal 0.2705 for the new I-optimal design and 0.2900 for the original I-optimal design.

\subsection{Four ingredients}

Scenario 5 involves four ingedients with availabilities $2.5 \mathrm{~kg}, 6 \mathrm{~kg}, 3 \mathrm{~kg}$ and $7 \mathrm{~kg}$. Every experimental run requires proportions of at least 0.2 for ingredient $1,0.1$ for ingredient $2,0.1$ for ingredient 3 , and 0.2 for 


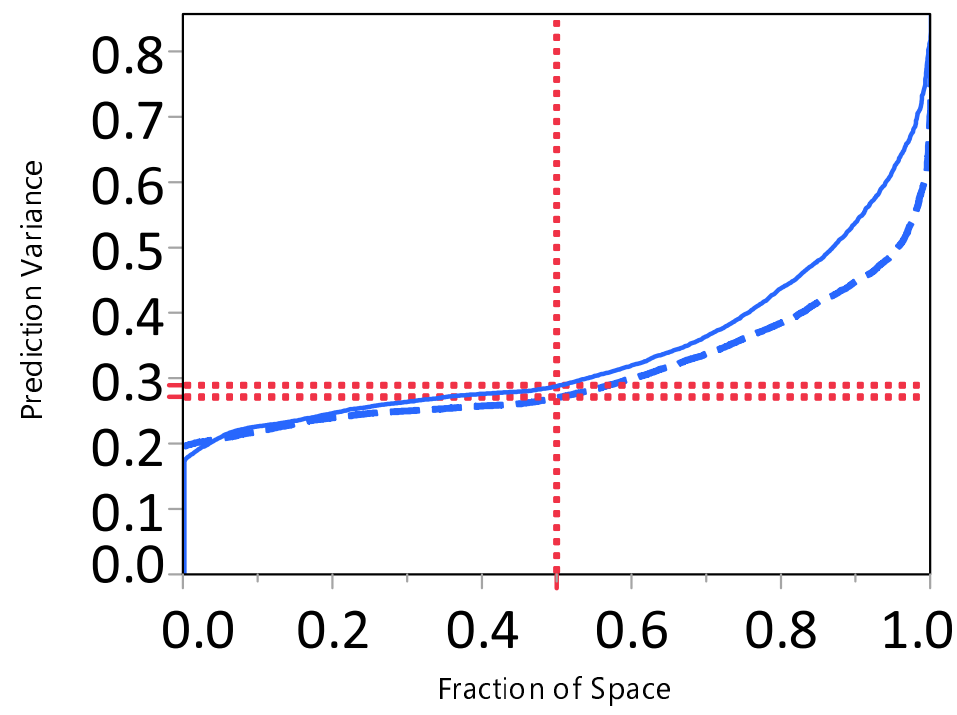

Figure 5: Fraction of design space plot comparing the original and the new I-optimal design for Scenario 4. The solid line represents the original I-optimal design, while the dashed line represents the new I-optimal design.

ingredient 4 .

The I-optimal design for the second-order Scheffé model in that scenario involves ten runs and is shown in Table 2. The table shows the design expressed in the original ingredients as well as in the pseudocomponents. The former representation is convenient because it allows us to verify that the ingredient availability constraints are satisfied, whereas the latter representation makes it easy to identify vertices of the experimental region, design points that lie on edges or faces of the experimental region, and interior points.

Remarkably, the design only involves three of the four vertices of the experimental region. The missing vertex is the point $\left(x_{1}, x_{2}, x_{3}, x_{4}\right)=(0.6,0.1,0.1,0.2)$, which corresponds to a mixture with the maximum proportion of $x_{1}$ possible and the minimum proportions of $x_{2}, x_{3}$ and $x_{4}$ possible. This vertex is missing in the I-optimal design because $x_{1}$ is the most scarce ingredient and, in addition, it has the largest lower bound for its proportion. In addition to the three vertices, the I-optimal design has four design points that lie on an edge of the experimental region, two points that lie on a face of the experimental region and one interior point. The I-optimality criterion value of the design is 1.0818 .

Note that the availabilities in Scenario 5 only just suffice to create a design with ten runs, which is the minimum required number of runs to estimate the second-order model in the four ingredients. Also, the ten runs are conducted at ten distinct design points, to ensure estimability of the second-order model, which involves ten parameters. Therefore, there are no degrees of freedom left to estimate the error variance.

A remarkable fact of the I-optimal design is that its maximum prediction variance is much larger than the average prediction variance. This is due to the fact that the design does not include a run at the vertex $\left(x_{1}, x_{2}, x_{3}, x_{4}\right)=(0.6,0.1,0.1,0.2)$, and, as a result of that, the prediction variance at that point is large (17.84) compared to the average variance of prediction (1.0818). This large prediction variance is a direct result from the limited availability of ingredient 1, which makes the use of design points involving large quantities of that ingredient impossible.

The large maximum prediction variance of the I-optimal design and the fact that its number of runs equals the number of model parameters are two weaknesses, which are due to the limited availability of ingredient 1. As a matter of fact, ingredient 1 is the only ingredient whose stock is entirely used by the I-optimal design. 
Table 2: I-optimal design involving four ingredients for Scenario 5, based on the original ingredient availabilities.

\begin{tabular}{r|rrrr|rrrr|c}
\hline \multirow{2}{*}{ Number } & \multicolumn{6}{|c|}{ Original proportion } & \multicolumn{5}{|c}{ Pseudocomponents } & \multirow{2}{*}{ Design point } \\
& $x_{1}$ & $x_{2}$ & $x_{3}$ & $x_{4}$ & $x_{1}^{*}$ & $x_{2}^{*}$ & $x_{3}^{*}$ & $x_{4}^{*}$ & \\
\hline 1 & 0.2 & 0.1 & 0.1 & 0.6 & 0 & 0 & 0 & 1 & Vertex \\
2 & 0.2 & 0.1 & 0.3 & 0.4 & 0 & 0 & 0.5 & 0.5 & Edge \\
3 & 0.2 & 0.1 & 0.5 & 0.2 & 0 & 0 & 1 & 0 & Vertex \\
4 & 0.2 & 0.3 & 0.1 & 0.4 & 0 & 0.5 & 0 & 0.5 & Edge \\
5 & 0.2 & 0.3 & 0.3 & 0.2 & 0 & 0.5 & 0.5 & 0 & Edge \\
6 & 0.2 & 0.5 & 0.1 & 0.2 & 0 & 1 & 0 & 0 & Vertex \\
7 & 0.25 & 0.1 & 0.1 & 0.55 & 0.125 & 0 & 0 & 0.875 & Edge \\
8 & 0.3 & 0.1 & 0.35 & 0.25 & 0.25 & 0 & 0.625 & 0.125 & Face \\
9 & 0.3 & 0.35 & 0.1 & 0.25 & 0.25 & 0.625 & 0 & 0.125 & Face \\
10 & 0.45 & 0.15 & 0.15 & 0.25 & 0.625 & 0.125 & 0.125 & 0.125 & Interior \\
\hline Total & 2.5 & 2.3 & 2.1 & 3.1 & & & & & 10 \\
\hline Availability & 2.5 & 6 & 3 & 7 & & & & & 18.5 \\
\hline
\end{tabular}

An experimenter facing such a situation might therefore consider acquiring some additional stock of the first ingredient. In order to decide on an appropriate amount of stock to acquire, we recommend a sensitivity study in which the total availability of the first ingredient is gradually increased to study the impact on the resulting I-optimal design, the corresponding average prediction variances and the corresponding maximum prediction variances. In case several ingredients have a limited stock, the sensitivity study should involve a gradual increase of the availabilities of more than one ingredient.

Suppose, as an illustration, that 2 additional kilograms of the first ingredient and 1.5 additional kilograms of the third ingredient are acquired, resulting in total availabilities of $4.5 \mathrm{~kg}$ for the first ingredient, $6 \mathrm{~kg}$ for the second ingredient, $4.5 \mathrm{~kg}$ for the third ingredient, and $7 \mathrm{~kg}$ for the fourth ingredient. The I-optimal design we obtained for the increased availabilities is shown in Table 3. The design consists of 14 distinct design points and 17 runs. Despite the substantially larger available stock of ingredient 1 , the new I-optimal design does not involve the vertex $\left(x_{1}, x_{2}, x_{3}, x_{4}\right)=(0.6,0.1,0.1,0.2)$. So, rather than using one run that uses the maximum amount of ingredient 1, it is better, from an I-optimality criterion point of view, to use design points which use a smaller proportion of ingredient 1 . The three other vertices of the design region are included in the design once. Ten runs are conducted at one of the edges of the experimental region, and the four final runs are performed at one of the experimental region's faces. The new I-optimal design requires $4.5 \mathrm{~kg}$ of the first ingredient, $3.6 \mathrm{~kg}$ of the second ingredient, $3.6 \mathrm{~kg}$ of the third ingredient, and $5.3 \mathrm{~kg}$ of the fourth ingredient. So, the design uses the complete additional stock of the first ingredient and some of the extra stock of the third ingredient.

The absence of the vertex $\left(x_{1}, x_{2}, x_{3}, x_{4}\right)=(0.6,0.1,0.1,0.2)$ in the design results in a larger prediction variance at that point when compared to other parts of the experimental region. When compared to the original I-optimal design, the new design, however, leads to a maximum prediction variance that is considerably smaller at that vertex. For the original design, the prediction variance amounts to 17.84 at that point, while it is 2.33 for the new design. The average variance of prediction is 0.3090 for the new I-optimal design, compared to 1.0818 for the original I-optimal design. Figure 6 compares the fraction of design space plots for the two designs in Scenario 5. Clearly, the new design (represented by the dashed line) performs much better than the original design (represented by the solid line). It is clear that the additional availability of certain ingredients has a major impact on the maximum, the median and the average prediction variance.

Unlike the I-optimal design in Table 2, the new I-optimal design in Table 3 allows estimation of the error variance, and, because three design points are used twice, even a lack-of-fit test. 
Table 3: I-optimal design involving four ingredients for Scenario 5, based on the new ingredient availabities.

\begin{tabular}{|c|c|c|c|c|c|c|c|c|c|}
\hline \multirow{2}{*}{ Number } & \multicolumn{4}{|c|}{ Original proportion } & \multicolumn{4}{|c|}{ Pseudocomponents } & \multirow{2}{*}{ Design point } \\
\hline & $x_{1}$ & $x_{2}$ & $x_{3}$ & $x_{4}$ & $x_{1}^{*}$ & $x_{2}^{*}$ & $x_{3}^{*}$ & $x_{4}^{*}$ & \\
\hline 1 & 0.2 & 0.1 & 0.1 & 0.6 & 0 & 0 & 0 & 1 & Vertex \\
\hline 2 & 0.2 & 0.1 & 0.5 & 0.2 & 0 & 0 & 1 & 0 & Vertex \\
\hline 3 & 0.2 & 0.5 & 0.1 & 0.2 & 0 & 1 & 0 & 0 & Vertex \\
\hline 4 & 0.2 & 0.1 & 0.3 & 0.4 & 0 & 0 & 0.5 & 0.5 & Edge \\
\hline 5 & 0.2 & 0.1 & 0.3 & 0.4 & 0 & 0 & 0.5 & 0.5 & Edge \\
\hline 6 & 0.2 & 0.3 & 0.1 & 0.4 & 0 & 0.5 & 0 & 0.5 & Edge \\
\hline 7 & 0.2 & 0.3 & 0.1 & 0.4 & 0 & 0.5 & 0 & 0.5 & Edge \\
\hline 8 & 0.2 & 0.3 & 0.3 & 0.2 & 0 & 0.5 & 0.5 & 0 & Edge \\
\hline 9 & 0.2 & 0.3 & 0.3 & 0.2 & 0 & 0.5 & 0.5 & 0 & Edge \\
\hline 10 & 0.35 & 0.1 & 0.1 & 0.45 & 0.375 & 0 & 0 & 0.625 & Edge \\
\hline 11 & 0.35 & 0.1 & 0.35 & 0.2 & 0.375 & 0 & 0.625 & 0 & Edge \\
\hline 12 & 0.35 & 0.35 & 0.1 & 0.2 & 0.375 & 0.625 & 0 & 0 & Edge \\
\hline 13 & 0.55 & 0.1 & 0.1 & 0.25 & 0.875 & 0 & 0 & 0.125 & Edge \\
\hline 14 & 0.2 & 0.25 & 0.25 & 0.3 & 0 & 0.375 & 0.375 & 0.25 & Face \\
\hline 15 & 0.3 & 0.1 & 0.25 & 0.35 & 0.25 & 0 & 0.375 & 0.375 & Face \\
\hline 16 & 0.3 & 0.25 & 0.1 & 0.35 & 0.25 & 0.375 & 0 & 0.375 & Face \\
\hline 17 & 0.3 & 0.25 & 0.25 & 0.2 & 0.25 & 0.375 & 0.375 & 0 & Face \\
\hline Total & 4.5 & 3.6 & 3.6 & 5.3 & & & & & \\
\hline Availability & 4.5 & 6 & 4.5 & 7 & & & & & \\
\hline
\end{tabular}

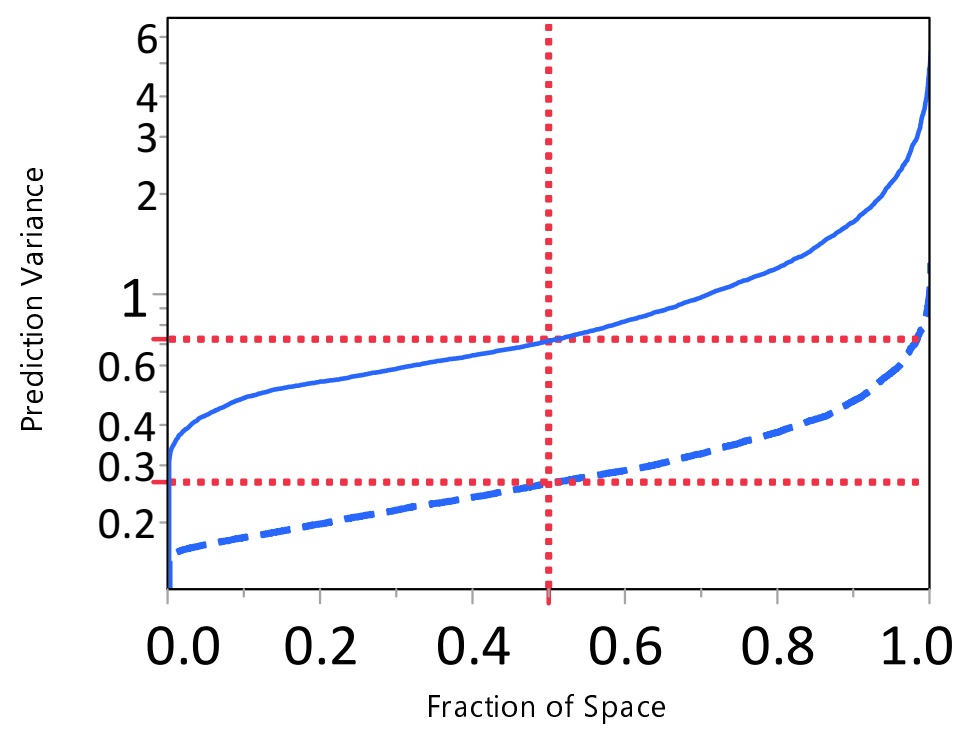

Figure 6: Fraction of design space plot comparing the original and the new I-optimal design for Scenario 5. The solid line represents the original I-optimal design, while the dashed line represents the new I-optimal design. 


\section{Summary}

In this article, we introduced a new type of problem in the field of optimal design of experiments, i.e. a problem involving limited availabilities of ingredients in a mixture. To solve instances of the new optimal design problem, we used a new variable neighborhood search algorithm. The algorithm has the advantage that it generally does not get stuck in local optima as often as simpler algorithms that are commonly used in optimal experimental design (such as point-exchange and coordinate-exchange algorithms). Moreover, the algorithm is more intuitive than genetic algorithms and simulated annealing, for instance.

We demonstrated the potential of the variable neighborhood search algorithm to generate I-optimal designs by means of several examples. The resulting optimal designs are very different from the optimal designs constructed in the literature under the assumption that there are no restrictions on the ingredient usage. For instance, the new designs often involve unequal replication of the different design points. Design points which use a large proportion of a scarce ingredient are generally replicated less frequently than points which use a small proportion of that ingredient. In some cases, experimenters may want to explore alternative designs that replicate the design points as evenly as possible across the experimental region. This may require sacrificing several runs involving a little bit of one scarce ingredient in exchange for one run that uses a lot of the scarce ingredient. A systematic exploration of this approach would be a useful topic for future research.

Another feature of optimal mixture designs in the presence of ingredient availability constraints is that the distribution of design points across the experimental region depends on the lower bounds of the ingredients' proportions. This is not the case in the absence of ingredient availability constraints, where the distribution of points is entirely driven by the shape of the experimental region and not by the exact location of the lower bounds on the proportions.

In this article, we focus on I-optimal designs because mixture experiments are usually meant to optimize one or more responses and precise predictions are essential to achieve this purpose. However, our variable neighborhood search algorithm can also be used to construct D-optimal designs for mixture experiments in the presence of availability constraints. On various occasions, we observed substantial differences between Dand I-optimal designs. The most striking difference is that the number of runs in the D-optimal designs was higher on various occasions than the number of runs in the I-optimal designs. Despite their lower number of runs, the I-optimal designs still produce smaller prediction variances than their D-optimal counterparts. In most cases, the D-optimal design perfomed poorer in terms of I-optimality than the I-optimal design performed in terms of D-optimality. This is in line with the results of Hardin and Sloane (1993) and Jones and Goos (2012). In one case, however, the performance of the I-optimal design in terms of the D-optimality criterion was much poorer than the performance of the D-optimal design in terms of the I-optimality criterion. This demonstrates that the presence of ingredient availability constraints results in a substantially different optimal design problem, where insights acquired in the absence of ingredient availability constraints may no longer apply. Readers who are interested in a detailed comparison of D-optimal and I-optimal designs in the presence of ingredient availability constraints are referred to Syafitri, Sartono and Goos (2014).

\section{References}

Atkinson, A. C., Donev, A. N. and Tobias, R. D. (2007). Optimum Experimental Designs, with SAS, Oxford: Oxford University Press.

Avanthay, C., Hertz, A. and Zufferey, N. (2003). A variable neighborhood search for graph coloring, European Journal of Operational Research 151: 379-388.

Caporossi, G. and Hansen, P. (2004). Variable neighborhood search for extremal graphs. 5. Three ways to automate finding conjectures, Discrete Mathematics 276: 81-94.

Cornell, J. A. (2002). Experiments with Mixtures: Designs, Models, and the Analysis of Mixture Data, New York: Wiley.

De Groot, M. (1970). Optimal Statistical Decisions, New York: McGraw Hill. 
De Ketelaere, B., Goos, P. and Brijs, K. (2011). Prespecified factor level combinations in the optimal design of mixture-process variable experiments, Food Quality and Preference 22: 661-670.

Fleszar, K. and Hindi, K. S. (2004). Solving the resource-constrained project scheduling problem by a variable neighbourhood search, European Journal of Operational Research 155: 402-413.

Garroi, J., Goos, P. and Sorensen, K. (2010). Hard multidimensional multiple choice knapsack problem, an empirical study, Computers \&S Operations Research 37: 172-181.

Goos, P. and Jones, B. (2011). Design of Experiments: A Case Study Approach, New York: Wiley.

Goos, P., Jones, B. and Syafitri, U. (2013). I-optimal mixture designs, Working paper 033, Faculty of Applied Economics, University of Antwerp.

Goos, P. and Syafitri, U. (2014). V-optimal mixture designs for the qth degree model, Chemometrics and Intelligent Laboratory Systems 136: 173-178.

Hardin, R. H. and Sloane, N. J. A. (1993). A new approach to construction of optimal designs, Journal of Statistical Planning and Inference 37: 339-369.

Jones, B. and Goos, P. (2012). I-optimal versus D-optimal split-plot response-surface designs, Journal of Quality Technology 44: 85-101.

Kellerer, H., Pferschy, U. and Pisinger, D. (2010). Knapsack Problems, Heidelberg: Springer.

Kurotori, I. (1966). Experiments with mixtures of components having lower bounds, Industrial Quality Control 22: 592-596.

Kytöjoki, J., Nuortio, T., Bräysy, O. and Gendreau, M. (2007). An efficient variable neighborhood search heuristic for very large scale vehicle routing problems, Computers and Operations Research 34: 2743-2757.

Laake, P. (1975). On the optimal allocation of observation in experiments with mixtures, Scandinavian Journal of Statistics 2: 153-157.

Lambrakis, D. (1968a). Experiments with mixtures: A generalization of the simplex-lattice design, Journal of Royal Statistical Society, Ser. B 30(1): 123-136.

Lambrakis, D. (1968b). Experiments with p-component mixtures, Journal of Royal Statistical Society, Ser. B 30(1): 137-144.

Liu, S. and Neudecker, H. (1995). A V-optimal design for Scheffé's polynomial model, Statistics and Probability Letters 23: 253-258.

Mladenović, N. and Hansen, P. (1997). Variable neighbourhood search, Computers and Operations Research 24: 1097-1100.

Mladenović, N., Petrović, J., Kovačević-Vujčić, V. and Čangalović, M. (2003). Solving spread spectrum radar polyphase code design problem by tabu search and variable neighbourhood search, European Journal of Operational Research 151: 389-399.

Piepel, G., Cooley, S. and Jones, B. (2005). Construction of a 21-component layered mixture experiment design using a new mixture coordinate-exchange algorithm, Quality Engineering 17: 579-594.

Sartono, B., Goos, P. and Schoen, E. (2015). Constructing general orthogonal fractional factorial split-plot designs, Technometrics 57: To appear.

Scheffé, H. (1958). Experiments with mixtures, Journal of the Royal Statistical Society, Ser. B 20: 344-360. 
Scheffé, H. (1963). The simplex-centroid design for experiments with mixtures, Journal of the Royal Statistical Society, Ser. B 25: 235-263.

Smith, W. (2005). Experimental Design for Formulation, Philadelphia : Siam.

Syafitri, U., Sartono, B. and Goos, P. (2014). Optimal design of mixture experiments in the presence of ingredient availability constraints, Working paper, Universiteit Antwerpen, Faculty of Applied Economics. 